\title{
Editorial: Special Issue in Privacy, Security and Trust for Mobile Systems
}

\author{
Kewei Sha · Zhengping Wu
}

Published online: 26 September 2013

C Springer Science+Business Media New York 2013

Recently, mobile devices, such as wireless sensors, smart tags, RFIDs, tablets, PDAs and smart phones, have become pervasive and attracted significant interest from academia, industry, and standardization organizations. Integrating the latest cloud computing technology, these mobile devices are playing a more and more important role in computing and communication systems. Millions of mobile applications have been developed in the last several years, which dramatically changes the way that people manage their daily life, for example, mobile payment and mobile social networks have changed our life significantly. When the storage, processing and communicating of many sensitive data have been migrated to mobile devices, privacy, security and trust become critical factors for the acceptance of mobile applications and maintaining a reliable mobile environment becomes an essential requirement. Several favorable characteristics of mobile and wireless devices, including portability, mobility, and sensitivity, further impose the challenge of security and privacy in these systems. Finally, the constrained hardware and software supports of those devices make it even more challenging to build a secure, privacy-preserving and trusted mobile system.

Despite recent advances, many research issues still remain in the design of secure, privacypreserving, or trust architectures, protocols, algorithms, services, and applications on mobile and wireless systems. For example, when mobile devices have more storage space, higher bandwidth, and better sensing capability, more sensitive information will be stored over there and more demanding application will be created. On the other hand, operating systems running on these devices are not as powerful and reliable as those on traditional computers. Both OS layer and higher-level layer protocols are expected to enhance the security and preserve the privacy of these mobile devices. With more mobile devices being used in online social networks and various ad-hoc network systems, novel trust models are essential for new applications. New cryptographic algorithms, intrusion detection and prevention mechanisms,

\footnotetext{
K. Sha $(\bigotimes)$

Department of Technology and Software Engineering, Oklahoma City University, 2501 N Blackwelder, Oklahoma City, OK 73106, USA e-mail:ksha@okcu.edu

Z. Wu

Computer Science and Engineering, University of Bridgeport, 126 Park Avenue,

Bridgeport, CT 06604, USA
} 
and integrity protection schemes are also needed when considering the special characteristics of mobile and wireless devices.

This special issue of Privacy, Security and Trust for Mobile Systems includes eight selected papers exploring several privacy-security-trust-related techniques, including privacypreserving protocols, privacy policy analysis mechanisms, integrity checking, new cryptographic algorithms, attack detection and prevention, applied in very different types of mobile devices and systems.

Three papers discuss privacy issues in mobile social networks, smart grid and cloud computing. Wu and etc.'s paper proposes a knowledge-based analysis framework to detect dynamic and hidden privacy policy conflicts for mobile social network; while Zeadally and etc.'s paper systematically examines potential privacy issues and their solutions in smart grid. One more paper from Wang and etc. examines a slight holomorphic signature mechanism to provide a fine-grained authentication and authorization to facilitate mobile service access in a cloud computing environment; meanwhile, the privacy of the cloud user is preserved based on the proposed mechanism.

Two papers focus on cipher techniques modified or customized to adapt to certain unique features in mobile systems, such as low power communication and RFID. Parakh and etc.'s paper develops a block cipher algorithm suitable for low power devices with a reduced complexity while keeping its security strength. Coluccia's paper explores a stream cipher to remove massive key exchange to fit mobile devices' capability.

Three papers explore alternative attack detection and prevention mechanisms for systems with high mobility, such as vehicular ad-hoc networks and mobile ad-hoc networks. Verma and etc.'s paper and Jia and etc.'s paper propose two prevention mechanisms for denial of service attack in vehicle and mobile ad-hoc networks respectively. Both present a simple yet effective mechanism to identify attacker nodes and mitigate their attacking traffic with minimum secrecy exchange and computation. Bulut and etc.'s paper explores a secure routing mechanism for an untrusted network environment.

At last, guest editors would like to take this opportunity to thank all the authors of this special issue for their excellent contributions, and reviewers, for their valuable comments and constructive suggestions. Springer publishing staff is also acknowledged for their professionalism and flexibility with the organization of this issue.

\section{Author Biographies}

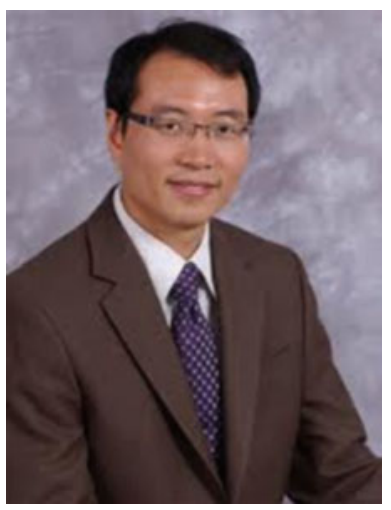

Kewei Sha is the Chair and Assistant Professor in the Department of Technology and Software Engineering at Oklahoma City University. He received Ph.D. in Computer Science from Wayne State University. His research interests include sensor networks, cyber-physical systems and mobile computing, data quality management, and network security and privacy. Dr. Sha authored one monograph, and published over 20 papers in various peer-reviewed prestigious conference proceedings and achieved journals. Dr. Sha has served as the secretary of Technical Committee on the Internet of the IEEE Computer Society (IEEECS TCI), a guest Editor at Wireless Personal Communications, a workshop general chair for ICCCN 2013, a workshop co-chair of MobiPST 2011 and 2012, a publicity chair for MobiPST 2013 and MCCTA 2013, a session chair in ICCCN and CollaborateCom, a member of editorial board in several journals, and a program committee member in numerous conferences. He is also a reviewer for numerous journals including IEEE TPDS, IEEE TC, ACM TAAS, Elsevier JPDC and so on. He is a member of IEEE and ACM. 


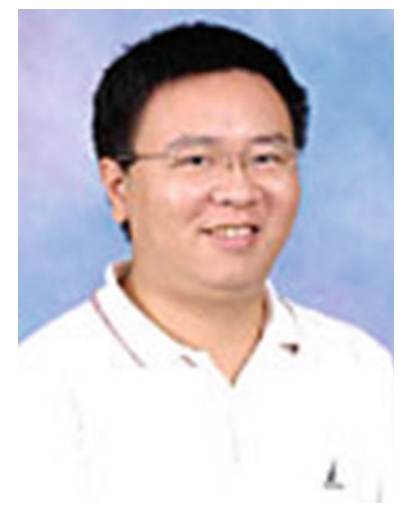

Zhengping Wu is an Assistant Professor of Computer Science and Engineering at the University of Bridgeport. He received his Ph.D. in computer science from the University of Virginia in 2008. He has authored or co-authored 7 book chapters and over 50 refereed research papers. His research interests include distributed systems, mobile computing, cloud computing, information security, medical informatics, and multimedia networks. 